\title{
Muscle Size, Strength, and Physical Function in Response to Augmented Calorie Delivery: A TARGET Sub-Study
}

Lee-anne S Chapple ( $\sim$ Lee-anne.chapple@adelaide.edu.au )

Royal Adelaide Hospital

Matthew J Summers

Royal Adelaide Hospital

Luke M Weinel

Royal Adelaide Hospital

Kylie Lange

The University of Adelaide

Woo Han Yang

Royal Adelaide Hospital

Adam M Deane

The University of Melbourne, Royal Melbourne Hospital

Marianne J Chapman

Royal Adelaide Hospital

\section{Research Article}

Keywords: Functional recovery, muscle mass, enteral nutrition, calories, intensive care, critical illness

Posted Date: February 24th, 2022

DOI: https://doi.org/10.21203/rs.3.rs-1367971/v1

License: (c) (1) This work is licensed under a Creative Commons Attribution 4.0 International License. Read Full License 


\section{Abstract \\ Background}

Critical illness leads to muscle wasting which may be attenuated with augmented calorie delivery. The Augmented versus Routine approach to Giving Energy Trial (TARGET) randomized 4000 patients to receive energy-dense $(1.5 \mathrm{kcal} / \mathrm{ml})$ or standard $(1.0 \mathrm{kcal} / \mathrm{ml})$ enteral nutrition. The aim of this sub-study was to evaluate whether augmented calorie delivery attenuates muscle loss and maintains strength and physical function when compared to routine care.

\section{Methods}

TARGET participants from a single participating ICU were eligible for enrolment in this sub-study if consent could be obtained. Ultrasound-derived muscle layer thickness (MLT) at three landmarks (quadriceps, forearm and mid-upper arm) and handgrip strength were measured at baseline and every 7 days until hospital discharge and at 3- and 6-months following randomization. Physical function was also assessed at 3- and 6-months using the 'get up and go' and 6-minute walk tests. Data are presented as mean \pm standard deviation.

\section{Results}

Eighty patients (1.5kcal: $n=38,58 \pm 14$ years, $60 \%$ male, APACHE II $20 \pm 7$ vs. $1.0 \mathrm{kcal}: \mathrm{n}=42,54 \pm 18$ years, $66 \%$ male, APACHE II $22 \pm 10$ ) were recruited. Although patients in the $1.5 \mathrm{kcal}$ group received more calories ( $2075 \pm 344$ vs $1325 \pm 313 \mathrm{kcal} /$ day; $\mathrm{P}<0.001)$, there was no significant difference in quadriceps MLT at any timepoint, including ICU discharge (primary outcome) (1.5kcal: $2.90 \pm 1.27$ vs $1.0 \mathrm{kcal}: 2.39 \pm$ $1.06 \mathrm{~cm} ; P=0.141)$, hospital discharge ( $2.47 \pm 1.03 \mathrm{vs} 2.10 \pm 1.08 \mathrm{~cm} ; P=0.227)$ or at 3 - and 6-months. Similar relationships were seen for forearm and mid-upper arm MLT and handgrip strength at all timepoints. Patients in the $1.5 \mathrm{kcal}$ group took less time to complete the 'get up and go' test $(6.66 \pm 1.33$ vs. $9.11 \pm 2.94$ secs; mean group difference $(95 \% \mathrm{Cl})-2.45(-4.35,-0.55) ; P=0.014)$, but there was no difference between groups at the 6-month follow-up. There were no differences in the distances walked in the 6-minute walk test at either 3-or 6-month timepoints.

\section{Conclusion}

Augmented calorie delivery compared to routine care did not attenuate loss of muscle size or strength during hospital admission or at 3- and 6-months following randomization. Patients receiving more calories had better physical function at 3-but not at 6-months when assessed using the get up and go test, but not the 6-minute walk test. 


\section{Introduction}

Critical illness leads to acute and rapid muscle wasting (1) in up to $80 \%$ of patients who receive mechanical ventilation for more than seven days (2). This muscle loss is significant, with studies using ultrasound-derived (US) rectus femoris cross-sectional area reporting skeletal muscle atrophy of almost $18 \%$ in the first 10 days of ICU admission (3). Structural measurements of muscle mass in ICU are strongly associated with function after ICU (4) and muscle atrophy is thought to contribute to 'ICUacquired weakness' that is associated with significant morbidity, slower weaning from mechanical ventilation, longer time to discharge, and substantially higher in-hospital costs (5). Patients experiencing ICU-acquired weakness have higher mortality rates one year after hospital discharge (5) and report persistent functional impairments and decreased quality of life $(6,7)$. Therefore, strategies aimed at attenuating muscle loss and improving functional recovery in these patients are critical.

It had been hypothesized that optimizing nutrition therapy to critically ill patients may help attenuate the observed muscle loss (8). A retrospective observational study in 33 critically ill patients with respiratory failure reported that change in skeletal muscle mass, determined by sequential lumbar CT scans was influenced by calorie delivery (9). Additionally, meeting calorie prescriptions early in ICU is associated with improved self-reported functional status three months post-injury (10). However, current standard practice provides the majority of critically ill patients with approximately $60 \%$ of their prescribed caloric needs ( 11 , 12) and there is an absence of data addressing the relationship between caloric delivery and muscle size, strength, or function.

Our group conducted The Augmented versus Routine Approach to Giving Energy Trial (TARGET) (13) which randomized almost 4,000 critically ill adults to receive energy-dense or routine enteral nutrition (EN) resulting in delivery of approximately $100 \%$ and $70 \%$ of recommended calorie targets, respectively (13). The intervention was not associated with improvements in 90-day mortality nor improved functional outcomes at 6-months quantified as capacity to return to work, disability, or societal participation (14). However, the functional outcomes included were self-reported and were measured at an extended duration following cessation of the trial intervention (at, or prior to, ICU discharge). Therefore, we aimed to determine whether the intervention attenuated acute skeletal muscle loss and maintained strength and function when measured objectively across the course of recovery when compared to routine care.

\section{Methods}

TARGET was a 4000-patient randomized, double-blind, pragmatic clinical trial conducted in 46 ICUs in Australia and New Zealand between June 21, 2016 and November 14, 2017. Detailed methods of the TARGET study have been previously published $(13,15)$. In short, mechanically ventilated critically ill patients were randomized to energy-dense EN ( $1.5 \mathrm{kcal}$ per milliliter) or routine EN (1.0 kcal per milliliter) at a dose of $1 \mathrm{ml}$ per kilogram of ideal body weight per hour, continuing for up to 28 days while the patient was in the ICU. TARGET was prospectively registered at ClinicalTrials.gov number, NCT02306746. 
This is an exploratory single-center sub-study to TARGET that was conducted at the Royal Adelaide Hospital (RAH), South Australia. It was approved by the Central Adelaide Local Health Network Human Research Ethics Committee.

\section{Patient recruitment:}

All patients enrolled into TARGET at the RAH were eligible for enrolment into this sub-study following an additional written informed consent obtained from the patient or their next of kin. Patients were eligible to be enrolled in TARGET and the sub-study if they were: 18 years of age or older; receiving invasive mechanical ventilation; about to commence EN or EN had commenced within the previous 12 hours; and were expected receive EN in the ICU beyond the calendar day after randomization. Patients were excluded from the sub-study if informed consent was unable to be obtained and the first ultrasound measure was unable to be conducted within 48 hours of randomization.

\section{Data collection:}

Data extracted from TARGET included: patient demographics (age, sex, admission diagnosis, Acute Physiology and Chronic Health Evaluation II (APACHE II) score); and nutrition data (baseline weight and body mass index (BMI), dietitian energy and protein prescription, daily energy and protein delivered during study period, duration of intervention received).

\section{Sub-study measurements:}

Ultrasound-derived muscle layer thickness (MLT) and handgrip strength were taken at baseline (within 48 hours of TARGET randomization) and conducted every 7 days ( \pm 72 hours) until hospital discharge, censored at 60 days. At 3- and 6-months ( \pm 30 days) after TARGET randomization, participants were invited to attend an in-hospital follow-up appointment at which the following measurements were conducted: ultrasound-derived MLT, handgrip strength, 24-hr dietary recall, 'get up and go' test, and sixminute walk test as detailed below. Patients who were unable to be contacted or could not physically attend the follow-up visit were classified as "lost to follow-up".

\section{Muscle layer thickness (MLT) measures using ultrasound (US):}

Ultrasound is established as a reliable method to quantify muscle thickness in critically ill patients $(3,16)$ and allows for repeated measures of muscle thickness in muscle groups such as the quadriceps, biceps and forearm that is representative of global muscle mass (17). A bedside ultrasound was performed by two trained operators (LC or LW) to determine the combined muscle thickness of two quadriceps muscles; the $M$. vastus intermedius and $M$. rectus femoris muscle as outlined previously $(16,18,19)$. In brief, two measurements of quadriceps MLT were taken: i) at the border between the lower third and upper twothirds between the Anterior Superior Iliac Spine (ASIS) and the upper pole of the patella, and ii) at the midpoint between the ASIS and the upper pole of the patella. The average of these two measurements 
were reported. MLT was also determined at the mid-arm (bicep brachii) between the humerus lateral epicondyle and superior lateral acromion border and at the anterior forearm at the mid-point between the tip of the olecranon and ulna styloid process. Landmarks were marked to ensure accuracy for follow-up measures, with all measures carried out on the right side unless unable due to injury or clinical intervention, to which the left side was used; the side measured was kept constant for each patient.

Ultrasound measures were conducted using a portable B-mode ultrasound (Sonosite X-Porte) using a 5$13 \mathrm{mHz}$ transducer by a trained operator blinded to the treatment allocation. All settings such as gain, focus and contrast were kept constant. Depth was set to $6 \mathrm{~cm}$ for quadriceps and mid-arm measures and $4.2 \mathrm{~cm}$ for forearm measures. The patient was positioned supine with legs relaxed lying flat in extension with toes facing the roof (where able). Liberal amounts of water-soluble transducer gel were used to minimize distortion, and the transducer held perpendicular to the skin surface to capture a transverse (cross-sectional) image. A still image was taken and saved with minimal transducer pressure, with the MLT calculated with onscreen calipers by the ultrasound operator. Two repeat measures were taken at each site, and a third taken if there was more than a 10\% discrepancy between the first two.

\section{Handgrip strength:}

In patients that were awake and able to follow commands, handgrip strength was measured every seven days during hospital admission with measurements conducted bilaterally in triplicate using a Jaymar digital hand dynamometer (20). The test was performed with the patient in upright position, shoulder in neutral rotation by the side of body and elbow flexed at 90 degrees. The patient was then directed to squeeze the dynamometer as hard as possible for three seconds. The highest measure for each hand was reported in kilograms. Cut-off values for diagnosing ICU-acquired weakness have been proposed to be $<11 \mathrm{~kg}$ for males, and $<7 \mathrm{~kg}$ for females (20).

\section{4-hour dietary recall:}

Oral nutrition intake was determined by a trained dietitian via a 24-hour recall. This data was entered into dietary analysis software Foodworks 8 (Xyris Pty Ltd., Brisbane, Australia) to quantify energy and macronutrient intake.

\section{Get up and go test}

From a seated position, patients were asked to rise from their chair, walk three meters, turn around and return to their original seated position with an average time calculated from three attempts (21).

\section{Six-minute walk test}

The 6-minute walk test was conducted according to the American Thoracic Society guidelines (22). Participants walked along a marked, $30 \mathrm{~m}$ flat, straight and hard-surfaced track for a period of six minutes at which time the distance travelled by the participant was recorded. The test was carried out unassisted with the participants' usual walking aids if required, with the test performed twice separated by a rest period, and the average of the two measures reported. 


\section{Statistics}

This was an exploratory study on the capacity for a randomized augmented calorie intervention to influence muscle size, and strength, and function. The primary analysis was the difference in the change in ultrasound-derived quadriceps MLT from baseline to ICU discharge between treatment groups. This was tested via analysis of covariance (ANCOVA) with treatment group and baseline MLT included as fixed effects. The first ultrasound measure available for each participant served as their baseline measure. Change in the repeated ultrasound measurements was performed using a mixed effects model with fixed effects for time, treatment group and the time by treatment group interaction, and a subject random effect to account for multiple measurements per participant. Differences between treatment groups on demographic and clinical characteristics were tested with independent samples t-tests and Mann-Whitney rank sum tests for continuous variables, and chi-square tests for categorical variables. At the time of protocol development there were insufficient data to determine a plausible and clinically significant difference in quadriceps MLT between groups; it was anticipated that a sample size of 100 patients (approximately 50 per group) would be eligible to participate over the expected 12-month recruitment period.

Reported measures used for analysis were categorized as baseline, day 7, ICU discharge and hospital discharge, censored at 60 days. ICU discharge and hospital discharge measurements included the closest available measurement to these timepoints. If ICU discharge and hospital discharge were within 72 hours of each other the same measurement was used. If ICU discharge or hospital discharge (or both) were within 72 hours of the day 7 measurement, the same measurement was used. If ICU discharge was within 72 hours of the baseline measure, the baseline measure was also used for ICU discharge. If MLT was > 6 $\mathrm{cm}$, i.e. out of depth field of view, this was coded as $6 \mathrm{~cm}$. Measurements were time-aligned, with time zero being cessation of TARGET EN. To describe differences in the mean trajectory between the ICU and post-ICU ward periods, a linear spline was included at time zero. The estimated mean trajectory for each group and $95 \%$ confidence intervals are shown graphically.

Exploratory analyses used multiple regression to examine the effect of baseline quadriceps MLT on quadriceps MLT at ICU discharge adjusting for baseline covariates of randomized treatment group, age, APACHE II score at ICU admission, BMI, and sex. A p-value of $<0.05$ was considered significant.

\section{Results}

\section{Enrolment}

Of the 169 patients enrolled into TARGET at the RAH, 80 (47\%) patients, or their next of kin, provided consent and were enrolled in this sub-study; 38 patients were randomized to the $1.5 \mathrm{kcal}$ group and 42 patients were randomized to the $1.0 \mathrm{kcal}$ group (Fig. 1). The primary reasons for exclusion from the substudy included being unable to obtain consent or the baseline measurements within 48 hours of randomization. Patient demographic data and clinical characteristics were well balanced between groups 
and are presented in Table 1. Of note, patients in the $1.5 \mathrm{kcal}$ group received a shorter duration of trial EN $(5.5(2.0,7.0)$ vs $8.0(4.0,12.0)$ days; mean group difference $(95 \% \mathrm{Cl})-2.0(-5.0,0.0) ; P=0.045)$. 
Table 1

Patient demographics and clinical characteristics

\begin{tabular}{|c|c|c|c|}
\hline & $\begin{array}{l}1.0 \mathrm{kcal} \\
(n=42)\end{array}$ & $\begin{array}{l}1.5 \mathrm{kcal} \\
(\mathrm{n}=38)\end{array}$ & $\begin{array}{l}\text { Group differences } \\
(95 \% \mathrm{Cl})\end{array}$ \\
\hline Age on ICU adm (years) & $\begin{array}{l}58.2 \pm \\
14.2\end{array}$ & $\begin{array}{l}53.5 \pm \\
17.6\end{array}$ & $-4.8(-11.8,2.3)$ \\
\hline Sex (male), n (\%) & $25(60 \%)$ & $25(66 \%)$ & \\
\hline APACHE II score on ICU adm & $19.8 \pm 6.9$ & $21.6 \pm 9.5$ & $1.8(-1.9,5.5)$ \\
\hline ICU admission diagnosis, $\mathrm{n}(\%)$ & $6(14 \%)$ & $6(16 \%)$ & \\
\hline Cardiovascular & $5(12 \%)$ & $7(18 \%)$ & \\
\hline Respiratory & $0(0 \%)$ & $0(0 \%)$ & \\
\hline Gastrointestinal & $15(36 \%)$ & $9(24 \%)$ & \\
\hline Neurological & $3(7 \%)$ & $3(8 \%)$ & \\
\hline Sepsis & $9(21 \%)$ & $11(29 \%)$ & \\
\hline Trauma & $4(10 \%)$ & $2(5 \%)$ & \\
\hline \multicolumn{4}{|l|}{ Other } \\
\hline Ideal body weight from height (kg) & $\begin{array}{l}65.5 \pm \\
10.2\end{array}$ & $66.6 \pm 9.6$ & $1.1(-3.3,5.5)$ \\
\hline Actual body weight (kg) & $\begin{array}{l}81.1 \pm \\
16.8\end{array}$ & $\begin{array}{l}84.7 \pm \\
16.3\end{array}$ & $3.6(-3.8,11.0)$ \\
\hline Body Mass Index $\left(\mathrm{kg} / \mathrm{m}^{2}\right)$ & $27.5 \pm 4.7$ & $28.7 \pm 6.4$ & $-0.005(-0.4,0.4)$ \\
\hline $\begin{array}{l}\text { Time from randomisation to commencing trial } \\
\text { nutrition (hours), median [IQR] }\end{array}$ & $\begin{array}{l}0.7(0.47 \\
2.18)\end{array}$ & $\begin{array}{l}0.9(0.48 \\
1.70)\end{array}$ & $-0.017(-0.4,0.3)$ \\
\hline $\begin{array}{l}\text { Duration of trial nutrition received (days), median } \\
\text { [IQR] }\end{array}$ & $\begin{array}{l}8.0(4.0 \\
12.0)\end{array}$ & $\begin{array}{l}5.5(2.0 \\
7.0)\end{array}$ & $-2.0(-5.0,0.0)$ \\
\hline Prescription as per treating clinician: & $\begin{array}{l}1968 \pm \\
368\end{array}$ & $\begin{array}{l}2018 \pm \\
344\end{array}$ & \\
\hline $\mathrm{kcal} / \mathrm{d}$ & $30.3 \pm 5$ & $30.7 \pm 5$ & \\
\hline $\mathrm{kcal} / \mathrm{kg} / \mathrm{d}$ & $91 \pm 16$ & $95 \pm 17$ & \\
\hline $\begin{array}{l}\text { g protein } / \mathrm{d} \\
\text { g protein } / \mathrm{kg} / \mathrm{d}\end{array}$ & $1.4 \pm 0.2$ & $1.4 \pm 0.2$ & \\
\hline
\end{tabular}




\begin{tabular}{|c|c|c|c|}
\hline & $\begin{array}{l}1.0 \mathrm{kcal} \\
(n=42)\end{array}$ & $\begin{array}{l}1.5 \mathrm{kcal} \\
(\mathrm{n}=38)\end{array}$ & $\begin{array}{l}\text { Group differences } \\
(95 \% \mathrm{Cl})\end{array}$ \\
\hline Total calories received: & $\begin{array}{l}1325 \pm \\
313\end{array}$ & $\begin{array}{l}2075 \pm \\
380\end{array}$ & \\
\hline From trial nutrition in ICU: & $20 \pm 4$ & $31 \pm 4$ & \\
\hline $\mathrm{kcal} / \mathrm{kg} \mathrm{IBW} / \mathrm{d}$ & $\begin{array}{l}1445 \pm \\
328\end{array}$ & $\begin{array}{l}2115 \pm \\
458\end{array}$ & \\
\hline $\begin{array}{l}\text { From all sources: } \\
\mathrm{kcal} / \mathrm{d} \\
\mathrm{kcal} / \mathrm{kg} \mathrm{IBW} / \mathrm{d}\end{array}$ & $22 \pm 4$ & $32 \pm 7$ & \\
\hline Total protein received, mean (SD): & $73 \pm 17$ & $77 \pm 14$ & \\
\hline & $\begin{array}{l}1.12 \pm \\
0.21\end{array}$ & $\begin{array}{l}1.17 \pm \\
0.16\end{array}$ & \\
\hline g/d & $73 \pm 17$ & $75 \pm 17$ & \\
\hline $\begin{array}{l}\text { g/kg IBW/d } \\
\text { From all sources: } \\
\text { g/d } \\
\text { g/kg IBW/d }\end{array}$ & $\begin{array}{l}1.12 \pm \\
0.21\end{array}$ & $\begin{array}{l}1.14 \pm \\
0.23\end{array}$ & \\
\hline Percent TARGET EN goal rate delivered & $85 \pm 16$ & $87 \pm 12$ & \\
\hline
\end{tabular}

\section{In-hospital measurements:}

\section{Ultrasound MLT measurements}

At all timepoints (baseline, day 7, ICU discharge, and hospital discharge) there were no significant differences in measurements of quadriceps, forearm or mid-upper arm MLT between patients in the 1.5 $\mathrm{kcal}$ and $1.0 \mathrm{kcal}$ groups (Table 2). A regression model accounting for baseline quadriceps MLT, illness severity (APACHE II), age, BMI, or sex showed no effect of treatment on quadriceps MLT at ICU discharge (Table 3). In addition, there was no relationship observed between calorie intake from trial EN and change in quadriceps MLT from baseline to ICU discharge (Fig. 2). 
Table 2

In-hospital measures of muscle size and strength

\begin{tabular}{|c|c|c|c|c|c|c|}
\hline & $\begin{array}{l}1.0 \mathrm{kcal} \\
(n=42)\end{array}$ & $\begin{array}{l}1.5 \mathrm{kcal} \\
(n=38)\end{array}$ & $\begin{array}{l}\text { Unadjusted } \\
\text { group difference } \\
(95 \% \mathrm{Cl})\end{array}$ & $\begin{array}{l}P \\
\text { value }\end{array}$ & $\begin{array}{l}\text { Baseline } \\
\text { adjusted group } \\
\text { difference ( } 95 \%\end{array}$ & $P$-value \\
\hline & $\begin{array}{l}\text { Mean } \pm \\
\text { SD }\end{array}$ & $\begin{array}{l}\text { Mean } \pm \\
\text { SD }\end{array}$ & & & & \\
\hline $\begin{array}{l}\text { Quadriceps } \\
\text { muscle layer }\end{array}$ & $2.86 \pm$ & $3.07 \pm$ & $0.21(-0.36$ & 0.470 & - & - \\
\hline thickness; cm & $=40$ & $=36$ & & 0.302 & $0.23(-0.14,0.59)$ & 0.213 \\
\hline At baseline & $2.38 \pm$ & $2.68 \pm$ & $0.86)$ & 0.141 & $0.42(-0.02,0.87)$ & 0.064 \\
\hline Day 7 & $=30$ & $=19$ & $0.51(-0.17$ & 0.227 & $0.31(-0.12,0.74)$ & 0.155 \\
\hline ICU discharge & $2.39 \pm$ & $2.90 \pm$ & & & & \\
\hline Hospital & $=29$ & $=19$ & $0.97)$ & & & \\
\hline & $\begin{array}{l}2.10 \pm \\
1.08, \mathrm{n} \\
=34\end{array}$ & $\begin{array}{l}2.47 \pm \\
1.03, \mathrm{n} \\
=20\end{array}$ & & & & \\
\hline Forearm muscle & $1.45 \pm$ & $1.40 \pm$ & $-0.05(-0.32$ & 0.731 & - & - \\
\hline & $=36$ & $=33$ & & 0.729 & $0.18(-0.18,0.55)$ & 0.319 \\
\hline At baseline & $1.32 \pm$ & $1.39 \pm$ & $0.49)$ & 0.172 & $0.26(0.00,0.51)$ & 0.047 \\
\hline Day 7 & $=22$ & $=17$ & $\begin{array}{l}0.23(-0.10, \\
0.56)\end{array}$ & 0.665 & $0.06(-0.18,0.31)$ & 0.598 \\
\hline ICU discharge & $1.22 \pm$ & $1.45 \pm$ & & & & \\
\hline Hospital & $\begin{array}{l}=43 \\
=23\end{array}$ & $=16$ & $0.32)$ & & & \\
\hline & $\begin{array}{l}1.26 \pm \\
0.44, \mathrm{n} \\
=29\end{array}$ & $\begin{array}{l}1.32 \pm \\
0.45, n \\
=20\end{array}$ & & & & \\
\hline Mid upper arm & $2.29 \pm$ & $2.00 \pm$ & $-0.30(-0.69$ & 0.133 & - & - \\
\hline thickness; cm & $=39$ & $=34$ & $-001(-0.42$ & 0.954 & $0.11(-0.25,0.47)$ & 0.542 \\
\hline At baseline & $1.81 \pm$ & $1.80 \pm$ & $0.39)$ & 0.208 & $0.36(0.06,0.66)$ & 0.020 \\
\hline Day 7 & $=26$ & $=17$ & $\begin{array}{l}0.24(-0.14 \\
0.61)\end{array}$ & 0.199 & $0.39(-0.01,0.78)$ & 0.055 \\
\hline ICU discharge & $1.68 \pm$ & $1.92 \pm$ & 0 & & & \\
\hline Hospital & $=27$ & $=16$ & $0.67)$ & & & \\
\hline & $\begin{array}{l}1.62 \pm \\
0.77, \mathrm{n} \\
=32\end{array}$ & $\begin{array}{l}1.88 \pm \\
0.61, n \\
=20\end{array}$ & & & & \\
\hline
\end{tabular}

ICU: Intensive Care Unit, SD: Standard deviation 


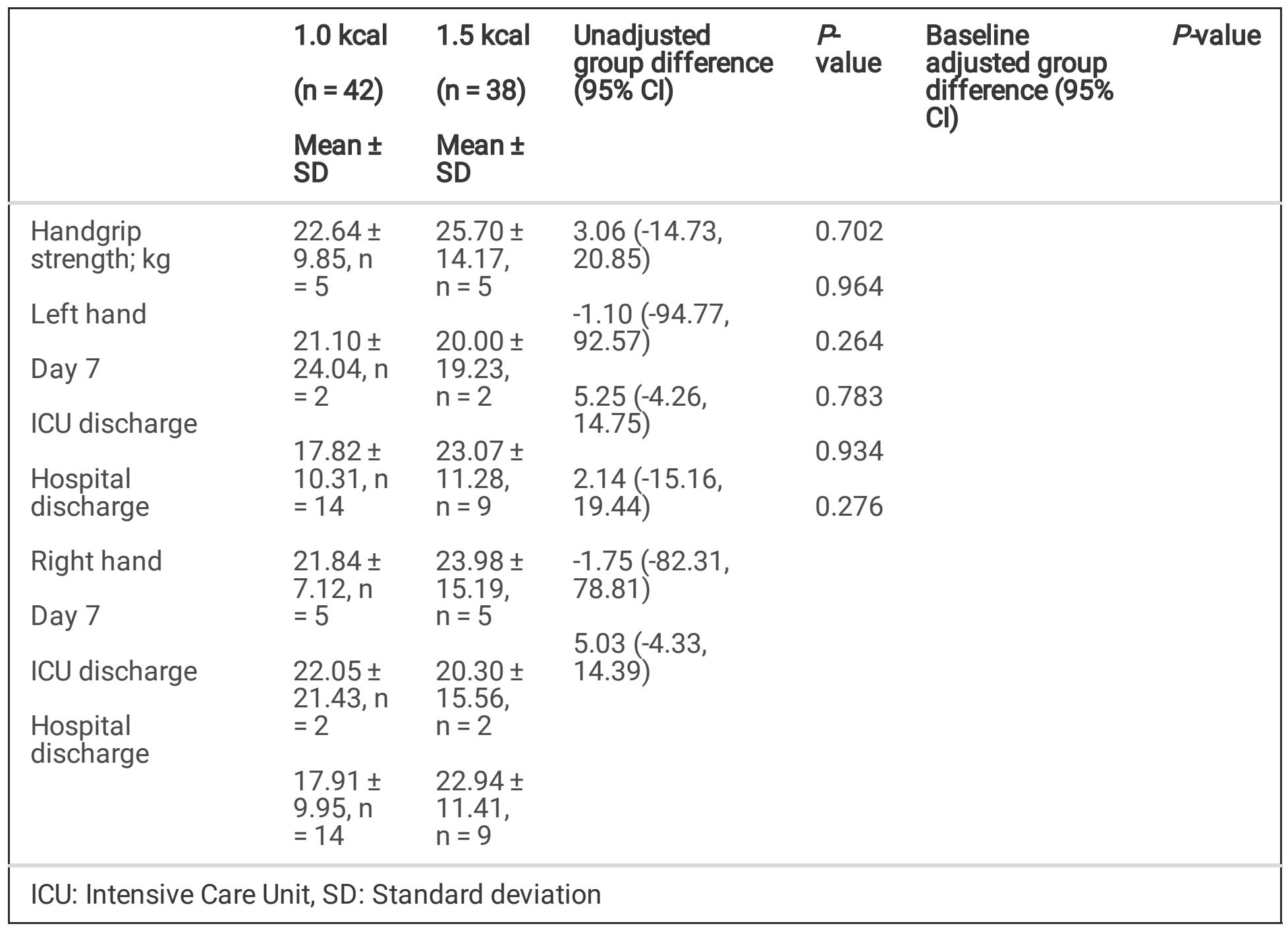

Table 3

Regression coefficients from model for quadriceps muscle layer thickness $(\mathrm{cm})$ at intensive care unit discharge

\begin{tabular}{|lllll|}
\hline Variables & \multicolumn{4}{c|}{ Effect estimate adjusted for all covariates } \\
\cline { 2 - 6 } & Effect & Standard error & $95 \%$ Confidence Interval & P-value \\
\hline Constant & -0.14 & 0.78 & $(-1.72,1.44)$ & 0.859 \\
\hline Baseline quadriceps MLT, cm & 0.65 & 0.12 & $(0.41,0.89)$ & 0.000 \\
\hline Treatment & 0.31 & 0.24 & $(-0.17,0.79)$ & 0.199 \\
\hline APACHE II & -0.01 & 0.02 & $(-0.05,0.03)$ & 0.507 \\
\hline Age (per 10 years) & -0.04 & 0.09 & $(-0.22,0.15)$ & 0.698 \\
\hline BMI, kg/m ${ }^{2}$ & 0.04 & 0.03 & $(-0.01,0.09)$ & 0.146 \\
\hline Sex & -0.07 & 0.25 & $(-0.57,0.43)$ & 0.767 \\
\hline $\begin{array}{l}\text { APACHE: Acute Physiology and Chronic Health Evaluation, BMI: Body Mass Index, MLT: Muscle layer } \\
\text { thickness }\end{array}$ & & & & \\
\hline
\end{tabular}




\section{Handgrip strength}

Handgrip strength did not differ between groups at any timepoint in hospital (day 7, ICU discharge, or hospital discharge). Few patients could provide a measurable handgrip strength within hospital, with only $5 \%$ patients (2 per group) being able to complete handgrip strength at ICU discharge, and only $29 \%$ of patients ( $\mathrm{n}=14$ and 9 , respectively) being able to complete handgrip strength at hospital discharge.

\section{3- and 6-month follow-up}

Thirty patients returned for the 3-month and 22 for the 6-month follow-ups. Nineteen patients returned for both the 3- and 6-month follow-up (Fig. 1).

\section{Ultrasound MLT measurements}

There were no significant differences in mean quadriceps, forearm or mid-upper arm MLT between 1.5 $\mathrm{kcal}$ and $1.0 \mathrm{kcal}$ groups at either 3- or 6-month follow-up (Table 4). 
Table 4

Muscle size, strength, and functional outcome measures at 3- and 6-months following trial randomization

\begin{tabular}{|c|c|c|c|c|c|c|c|}
\hline & & \multirow{2}{*}{$\begin{array}{l}1.0 \text { kcal } \\
\text { Mean } \pm \\
\text { SD, n }\end{array}$} & \multirow{2}{*}{$\begin{array}{l}1.5 \mathrm{kcal} \\
\text { Mean } \pm \\
\text { SD, n }\end{array}$} & \multicolumn{2}{|c|}{$\begin{array}{l}\text { Unadjusted Group } \\
\text { difference }\end{array}$} & \multicolumn{2}{|c|}{$\begin{array}{l}\text { Baseline adjusted } \\
\text { group difference }\end{array}$} \\
\hline & & & & $\begin{array}{l}\text { Mean } \\
(95 \% \mathrm{Cl})\end{array}$ & $\begin{array}{l}\mathrm{P}- \\
\text { value }\end{array}$ & $\begin{array}{l}\text { Mean }(95 \% \\
\mathrm{Cl})\end{array}$ & $\begin{array}{l}\mathrm{P} \text { - } \\
\text { value }\end{array}$ \\
\hline \multirow[t]{2}{*}{$\begin{array}{l}\text { Quadriceps muscle } \\
\text { layer thickness, cm }\end{array}$} & $\begin{array}{l}3 \\
\text { mo }\end{array}$ & $\begin{array}{l}2.70 \pm \\
1.46, n= \\
15\end{array}$ & $\begin{array}{l}3.26 \pm \\
0.95, n= \\
18\end{array}$ & $\begin{array}{l}0.57 \\
(-0.29 \\
1.43)\end{array}$ & 0.188 & $\begin{array}{l}0.31(-0.55 \\
1.17)\end{array}$ & 0.468 \\
\hline & $\begin{array}{l}6 \\
\text { mo }\end{array}$ & $\begin{array}{l}3.64 \pm \\
1.14, n= \\
8\end{array}$ & $\begin{array}{l}3.37 \pm \\
1.17, \mathrm{n}= \\
13\end{array}$ & $\begin{array}{l}-0.27 \\
(-1.36 \\
0.82)\end{array}$ & 0.610 & $\begin{array}{l}-0.33 \\
(-1.39,0.74)\end{array}$ & 0.528 \\
\hline \multirow[t]{2}{*}{$\begin{array}{l}\text { Forearm muscle layer } \\
\text { thickness, cm }\end{array}$} & $\begin{array}{l}3 \\
\text { mo }\end{array}$ & $\begin{array}{l}1.30 \pm \\
0.63, n= \\
12\end{array}$ & $\begin{array}{l}1.66 \pm \\
0.72, n= \\
14\end{array}$ & $\begin{array}{l}0.36 \\
(-0.20 \\
0.91)\end{array}$ & 0.195 & $\begin{array}{l}0.15(-0.38 \\
0.68)\end{array}$ & 0.557 \\
\hline & $\begin{array}{l}6 \\
\text { mo }\end{array}$ & $\begin{array}{l}1.73 \pm \\
0.90, n= \\
9\end{array}$ & $\begin{array}{l}1.40 \pm \\
0.61, \mathrm{n}= \\
11\end{array}$ & $\begin{array}{l}-0.33 \\
(-1.04 \\
0.38)\end{array}$ & 0.347 & $\begin{array}{l}-0.48 \\
(-1.25 \\
0.28)\end{array}$ & 0.198 \\
\hline \multirow[t]{2}{*}{$\begin{array}{l}\text { Mid upper arm } \\
\text { muscle layer } \\
\text { thickness, cm }\end{array}$} & $\begin{array}{l}3 \\
\text { mo }\end{array}$ & $\begin{array}{l}2.01 \pm \\
0.84, \mathrm{n}= \\
11\end{array}$ & $\begin{array}{l}2.16 \pm \\
1.02, n= \\
16\end{array}$ & $\begin{array}{l}0.14 \\
(-0.63 \\
0.91)\end{array}$ & 0.705 & $\begin{array}{l}0.04(-0.65 \\
0.73)\end{array}$ & 0.900 \\
\hline & $\begin{array}{l}6 \\
\text { mo }\end{array}$ & $\begin{array}{l}2.24 \pm \\
0.83, n= \\
10\end{array}$ & $\begin{array}{l}2.12 \pm \\
0.82, \mathrm{n}= \\
11\end{array}$ & $\begin{array}{l}-0.12 \\
(-0.88 \\
0.63)\end{array}$ & 0.737 & $\begin{array}{l}0.15(-0.46 \\
0.75)\end{array}$ & 0.619 \\
\hline \multirow[t]{2}{*}{$\begin{array}{l}\text { Handgrip strength, } \\
\text { Left side; kg }\end{array}$} & $\begin{array}{l}3 \\
\text { mo }\end{array}$ & $\begin{array}{l}28.15 \pm \\
12.70, \mathrm{n} \\
=12\end{array}$ & $\begin{array}{l}25.06 \pm \\
12.34, \mathrm{n} \\
=12\end{array}$ & $\begin{array}{l}-3.09 \\
(-13.69 \\
7.51)\end{array}$ & 0.551 & & \\
\hline & $\begin{array}{l}6 \\
\text { mo }\end{array}$ & $\begin{array}{l}34.23 \pm \\
13.27, \mathrm{n} \\
=9\end{array}$ & $\begin{array}{l}24.46 \pm \\
15.55, \mathrm{n} \\
=10\end{array}$ & $\begin{array}{l}-9.77 \\
(-23.85 \\
4.30)\end{array}$ & 0.161 & & \\
\hline \multirow[t]{2}{*}{$\begin{array}{l}\text { Handgrip strength, } \\
\text { Right side; kg }\end{array}$} & $\begin{array}{l}3 \\
\text { mo }\end{array}$ & $\begin{array}{l}31.96 \pm \\
12.67, \mathrm{n} \\
=12\end{array}$ & $\begin{array}{l}28.11 \pm \\
12.94, \mathrm{n} \\
=11\end{array}$ & $\begin{array}{l}-3.85 \\
(-14.96 \\
7.26)\end{array}$ & 0.479 & & \\
\hline & $\begin{array}{l}6 \\
\text { mo }\end{array}$ & $\begin{array}{l}36.11 \pm \\
12.36, \mathrm{n} \\
=9\end{array}$ & $\begin{array}{l}31.27 \pm \\
15.95, \mathrm{n} \\
=9\end{array}$ & $\begin{array}{l}-4.84 \\
(-19.11 \\
9.42)\end{array}$ & 0.482 & & \\
\hline \multirow[t]{2}{*}{$\begin{array}{l}\text { Get up and go; } \\
\text { seconds }\end{array}$} & $\begin{array}{l}3 \\
\text { mo }\end{array}$ & $\begin{array}{l}9.11 \pm \\
2.94, \mathrm{n}= \\
10\end{array}$ & $\begin{array}{l}6.66 \pm \\
1.33, n= \\
13\end{array}$ & $\begin{array}{l}-2.45 \\
(-4.35 \\
-0.55)\end{array}$ & 0.014 & & \\
\hline & $\begin{array}{l}6 \\
\text { mo }\end{array}$ & $\begin{array}{l}8.10 \pm \\
2.00, n= \\
9\end{array}$ & $\begin{array}{l}8.05 \pm \\
4.08, n= \\
8\end{array}$ & $\begin{array}{l}-0.05 \\
(-3.31 \\
3.21)\end{array}$ & 0.974 & & \\
\hline
\end{tabular}




\begin{tabular}{|c|c|c|c|c|c|c|}
\hline \multirow[b]{2}{*}{$\begin{array}{l}\text { Six-minute walk test; } \\
\text { metres }\end{array}$} & \multirow[b]{2}{*}{$\begin{array}{l}3 \\
\text { mo }\end{array}$} & \multirow{2}{*}{$\begin{array}{l}1.0 \mathrm{kcal} \\
\\
432.7 \pm \\
93.8, \mathrm{n}= \\
9\end{array}$} & \multirow{2}{*}{$\begin{array}{l}1.5 \mathrm{kcal} \\
\\
486.3 \pm \\
100.3, \mathrm{n} \\
=13\end{array}$} & \multicolumn{2}{|c|}{$\begin{array}{l}\text { Unadjusted Group } \\
\text { difference }\end{array}$} & \multirow[t]{2}{*}{$\begin{array}{l}\text { Baseline adjusted } \\
\text { group difference }\end{array}$} \\
\hline & & & & $\begin{array}{l}53.6 \\
(-34.8 \\
142.0)\end{array}$ & 0.220 & \\
\hline & $\begin{array}{l}6 \\
\text { mo }\end{array}$ & $\begin{array}{l}530.3 \pm \\
249.8, n \\
=10\end{array}$ & $\begin{array}{l}463.9 \pm \\
118.2, \mathrm{n} \\
=8\end{array}$ & $\begin{array}{l}-66.4 \\
(-270.6 \\
137.7)\end{array}$ & 0.500 & \\
\hline \multirow[t]{2}{*}{$\begin{array}{l}\text { Energy intake from } \\
24 \mathrm{~h} \text { recall, kcal }\end{array}$} & $\begin{array}{l}3 \\
\text { mo }\end{array}$ & $\begin{array}{l}2055 \pm \\
683, n= \\
10\end{array}$ & $\begin{array}{l}2551 \pm \\
1132, n= \\
14\end{array}$ & $\begin{array}{l}497(-339 \\
1333)\end{array}$ & 0.230 & \\
\hline & $\begin{array}{l}6 \\
\text { mo }\end{array}$ & $\begin{array}{l}2439 \pm \\
786, n= \\
10\end{array}$ & $\begin{array}{l}1599 \pm \\
704, n= \\
11\end{array}$ & $\begin{array}{l}-840 \\
(-1520 \\
-160)\end{array}$ & 0.018 & \\
\hline \multirow[t]{2}{*}{$\begin{array}{l}\text { Protein intake from } \\
24 \mathrm{~h} \text { recall, g }\end{array}$} & $\begin{array}{l}3 \\
\text { mo }\end{array}$ & $\begin{array}{l}89.8 \pm \\
35.8, n= \\
10\end{array}$ & $\begin{array}{l}116.8 \pm \\
61.1, \mathrm{n}= \\
14\end{array}$ & $\begin{array}{l}27.0 \\
(-17.9 \\
71.8)\end{array}$ & 0.225 & \\
\hline & $\begin{array}{l}6 \\
\text { mo }\end{array}$ & $\begin{array}{l}113.6 \pm \\
49.1, \mathrm{n}= \\
10\end{array}$ & $\begin{array}{l}68.3 \pm \\
27.0, n= \\
11\end{array}$ & $\begin{array}{l}-45.3 \\
(-81.0 \\
-9.6)\end{array}$ & 0.016 & \\
\hline \multirow[t]{2}{*}{$\begin{array}{l}\text { Fat intake from } 24 \mathrm{~h} \\
\text { recall, g }\end{array}$} & $\begin{array}{l}3 \\
\text { mo }\end{array}$ & $\begin{array}{l}68.0 \pm \\
28.8, n= \\
10\end{array}$ & $\begin{array}{l}94.5 \pm \\
54.8, n= \\
14\end{array}$ & $\begin{array}{l}26.5 \\
(-12.9 \\
66.0)\end{array}$ & 0.177 & \\
\hline & $\begin{array}{l}6 \\
\text { mo }\end{array}$ & $\begin{array}{l}101.0 \pm \\
42.4, n= \\
10\end{array}$ & $\begin{array}{l}62.0 \pm \\
36.3, n= \\
11\end{array}$ & $\begin{array}{l}-39.0 \\
(-74.9 \\
-3.1)\end{array}$ & 0.035 & \\
\hline \multirow[t]{2}{*}{$\begin{array}{l}\text { Carbohydrate intake } \\
\text { from } 24 \mathrm{~h} \text { recall, g }\end{array}$} & $\begin{array}{l}3 \\
\text { mo }\end{array}$ & $\begin{array}{l}238.0 \pm \\
104.3, \mathrm{n} \\
=10\end{array}$ & $\begin{array}{l}284.7 \pm \\
139.5, \mathrm{n} \\
=14\end{array}$ & $\begin{array}{l}46.7 \\
(-61.8 \\
155.1)\end{array}$ & 0.382 & \\
\hline & $\begin{array}{l}6 \\
\text { mo }\end{array}$ & $\begin{array}{l}247.6 \pm \\
111.8, n \\
=10\end{array}$ & $\begin{array}{l}162.3 \pm \\
83.6, n= \\
11\end{array}$ & $\begin{array}{l}-85.3 \\
(-174.9 \\
4.3)\end{array}$ & 0.061 & \\
\hline
\end{tabular}

\section{Handgrip strength}

There was no difference between $1.5 \mathrm{kcal}$ and $1.0 \mathrm{kcal}$ groups in handgrip strength taken on either the right- or left-hand side at 3- or 6-month follow-up (Table 4).

\section{4-hour dietary recall}

There was no significant difference in calorie intake, nor any of the macronutrient intakes, at 3-month follow-up between the groups (Table 4). At 6-month follow-up, patients in the $1.5 \mathrm{kcal}$ group reported lower calorie intakes compared to the $1.0 \mathrm{kcal}$ group (1599 \pm 704 vs $2439 \pm 786 \mathrm{kcal}$; mean group difference $(95 \% \mathrm{Cl})-840(-1520,-160) ; P=0.018)$ and this was driven by higher protein and fat intakes in 
the $1.0 \mathrm{kcal}$ group; however, participant numbers were small at this timepoint (10 and 11 patients, respectively).

\section{Get up and go test}

Patients in the $1.5 \mathrm{kcal}$ group took less time to complete the get up and go test at 3-months (greater time $=$ worse function) (1.5 kcal: $n=10,6.66 \pm 1.33$ secs vs $1.0 \mathrm{kcal}: \mathrm{n}=13,9.11 \pm 2.94$ secs; mean group difference $(95 \% \mathrm{Cl})-2.45(-4.35,-0.55) ; P=0.014)$, but there was no difference between groups at the 6month follow-up (Table 4).

\section{Six-minute walk test}

The distance walked in 6-minutes at both 3- and 6-month follow-ups did not differ between groups (Table 4).

\section{Discussion}

This study quantified change in muscle size, strength, and functional recovery in critically ill patients randomized to augmented calorie delivery compared to routine care. We observed that augmented calorie delivery had no quantifiable effect on quadriceps, forearm, or mid-upper arm muscle thickness or handgrip strength during the hospital admission. Furthermore, augmented calorie delivery in ICU did not influence muscle size or strength at 3-and 6-months after randomization. While patients in the augmented calorie group had a faster get up and go time at 3-months, this difference did not persist to 6months, nor did the distance walked in six minutes differ between groups at either of these timepoints.

The absence of reporting of muscle size, strength or function in ICU nutrition studies has previously been highlighted by our group in a systematic review; in 73 RCTs of nutrition interventions identified, only two included a measure of physical function as a primary outcome and $10 \%$ as a secondary or tertiary outcome (23). Of these studies, three compared early supplemental parenteral nutrition (PN) to routine care, the largest of which reported reduced muscle and fat loss (using Subjective Global Assessment and mid-arm muscle circumference) with supplemental PN in 1372 patients (24). Two pilot RCTs showed no difference in physical function using the ICU mobility scale at hospital discharge or handgrip strength at ICU or hospital discharge, (25) and non-significant improvements in handgrip strength at ICU and hospital discharge, and 6-minute walk test score and Barthel Index at hospital discharge (26). However, these studies did not provide augmented calories alone, with the trial interventions also providing augmented protein, which differs to our study in which protein delivery was similar between groups. In addition, a retrospective analysis of prospectively collected data including 302 patients alive at follow-up reported that meeting calorie targets in the first week of ICU was associated with a higher Short Form-36 physical component score at 3-months, but not 6-months; however, in this analysis protein intake was also not controlled for (10). 
Our study reports a number of measures of muscle size, strength and function that have previously been reported outside of a calorie intervention. Quadriceps MLT has been previously assessed in a study in 16 critically ill patients where a mean of $1.68 \mathrm{~cm}$ was observed on day 16 of ICU admission (27). This is substantially lower than the quadriceps MLT measurements observed in our study, even at hospital discharge, despite both populations being of a similar age and BMI. These differing results may be explained by differences in ultrasound methodology, with recognition that significant variations in measurement technique occur (28) which may affect results. In our study, mean handgrip strength was greater than $20 \mathrm{~kg}$ at both ICU discharge and on day 7. This is markedly higher than we have previously recorded in a cohort of patients with a prolonged ICU stay ( $\geq 5$ days) $(12.6 \mathrm{~kg})(29)$. At hospital discharge, the observed handgrip strength in our study $(\sim 17-23 \mathrm{~kg})$ is comparable to that reported previously (mean handgrip strength 23.2 (IQR 13.6-32.3) kg) (30); however, at 3-months our population had a higher handgrip strength $(\sim 25-28 \mathrm{~kg})$ than reported in other studies (20.4 (IQR 9.1-30.6) kg) (31). In relation to function, at 3-month follow-up our cohort walked a mean distance in six minutes of 486 and $432 \mathrm{~m}$ in the 1.5 and $1.0 \mathrm{kcal}$ groups, respectively, and 463 and $530 \mathrm{~m}$ at 6-month follow-up. In patients with acute respiratory distress syndrome, shorter median distances of $281 \mathrm{~m}$ at 3-months and $422 \mathrm{~m}$ at 12-months have been reported (6). Reasons for differences in muscle size, strength and function observed in our sub-study when compared to previous literature, particularly at post-hospital follow-up periods, is potentially related to the differing patient populations.

While calorie delivery may have no impact on muscle size, strength or functional recovery, it may also be that the duration of intervention is important. In this sub-study, patients had a short median duration of trial EN (5.5-8 days), which may reduce the potential effect of calorie delivery. Studies in non-ICU populations have shown benefit from nutritional interventions provided over longer durations. For example, Schuetz et al compared individualized nutrition support to routine care in 2088 non-critically ill hospitalized patients at nutritional risk, observing that patients receiving the study intervention over their entire hospital duration had improved calorie and protein adequacy, reduced mortality, improved physical recovery, and improved quality of life (32). Given poor nutrition intake post-ICU has been reported $(33,34)$, the effect of calorie delivery over the entire hospital stay on functional recovery needs to be considered.

In this study, only calorie delivery was significantly different between the study groups, while protein intake was kept consistent, yet with both groups receiving lower protein amounts $(1.12-1.17 \mathrm{~g} / \mathrm{kg}$ IBW/day) than recommended in international guidelines $(1.2-2.0 \mathrm{~g} / \mathrm{kg} /$ day $)(35,36)$. In health, muscle maintenance is dependent on amino acid availability (37), and hence calorie delivery alone, without augmented protein doses, may not be sufficient to attenuate muscle loss and improve functional recovery. It is possible that at a higher protein dose, increased calorie delivery may have had an effect, or that muscle mass and function respond to increased protein alone. To date, the literature on augmented protein dose to attenuate muscle loss is conflicting. Two small RCTs report a positive effect of protein dose on attenuating muscle; in 119 patients, higher intravenous doses of protein showed greater amelioration of ultrasound-derived muscle loss and a trend towards improved handgrip strength (19), and in 60 patients an augmented calorie and protein intervention attenuated muscle loss by ICU discharge (38). Meanwhile, more recent studies have reported no effect on muscle size using an $\beta$-Hydroxy $\beta$ - 
methylbutyric acid $(\mathrm{HMB})$ intervention $(39,40)$ or continuous vs intermittent EN $(41)$. The role of protein dose on muscle size in critical illness needs further investigation.

Both the main TARGET study and this sub-study used highly rigorous methodology, with TARGET randomly assigning patients to EN interventions in a double-blind fashion (13), and the sub-study using objective measures of muscle size, strength, and function which have been validated in the elderly or critically ill patients $(20-22,42)$. Duration of feeding differed between the study groups which may have influenced outcomes. A number of previous studies have used MLT as opposed to cross-sectional area (CSA) (28). While CSA has been shown to provide a more reliable indicator of muscle wasting (43), MLT may have greater translation into the clinical setting due to the ability to obtain results at the bedside (42), and may be easier to identify than CSA, particularly in the setting of muscle wasting. Further, all measures were conducted by two investigators only, and while interrater reliability was not assessed, consistency was ensured through rigorous training.

\section{Conclusion}

Delivering greater amounts of calories to critically ill patients whilst in ICU may not influence muscle size or strength during the hospital admission or at 3- or 6-months. Augmented calorie delivery may hasten aspects of functional recovery, but this requires confirmation in a larger trial.

\section{Declarations}

\section{Ethics approval and consent to participate:}

This TARGET sub-study was approved by the Central Adelaide Local Health Network Human Research Ethics Committee with local governance approval. Written informed consent was obtained from the patient or their next of kin prior to sub-study enrolment.

\section{Consent for publication:}

Written informed consent was obtained from all individual patients or their legal next of kin for publication of the data included within this manuscript.

\section{Data sharing agreement:}

Non-identifiable data that underlie the results reported in this trial will be made available three years following publication and ending five years after publication of the main manuscript. Availability will only be made to independent researchers who provide a written proposal for data evaluation that is judged to be methodologically sound by an independent committee approved by the sponsor. Proposals should be directed to lee-anne.chapple@adelaide.edu.au. If the proposal is approved applicants will be required to sign a data access agreement and will remain responsible for all costs incurred.

\section{Competing interests:}


All authors have no competing interests to declare.

\section{Funding:}

This study was funded by a 2015 Royal Adelaide Hospital Clinical Project Grant. The funders had no role in the study conduct, interpretation or manuscript preparation. LS Chapple has been supported by a European Society for Clinical Nutrition and Metabolism Research Fellowship (2018) and a National Health and Medical Research Council Early Career Fellowship (2019-2022).

\section{Authors' contributions:}

LSC, AMD, and MJC made substantial contributions to the conception or design of the work. LSC, MJS, LMW, and WHY were responsible for the acquisition and analysis of the work. LSC, MJS, LMW, KL, AMD, and MJC were responsible for interpretation of data for the work. LSC, LMW, and MJS drafted the work and all other authors revised it critically for important intellectual content. All authors approved the submitted version and have agreed to be personally accountable their own contributions and to ensure that questions related to the accuracy or integrity of any part of the work, even ones in which they were not personally involved, are appropriately investigated, resolved, and the resolution documented in the literature.

\section{Acknowledgements:}

The authors would like to acknowledge Caroline Cousins for her assistance with data entry and checking.

\section{References}

1. Llano-Diez M, Renaud G, Andersson M, Marrero HG, Cacciani N, Engquist H, et al. Mechanisms underlying ICU muscle wasting and effects of passive mechanical loading. Crit Care. 2012;16(5):R209.

2. Koukourikos K, Tsaloglidou A, Kourkouta L. Muscle atrophy in intensive care unit patients. Acta Inform Med. 2014;22(6):406-10.

3. Puthucheary ZA, Rawal J, McPhail M, Connolly B, Ratnayake G, Chan P, et al. Acute skeletal muscle wasting in critical illness. JAMA. 2013;310(15):1591-600.

4. Seymour JM, Ward K, Sidhu PS, Puthucheary Z, Steier J, Jolley CJ, et al. Ultrasound measurement of rectus femoris cross-sectional area and the relationship with quadriceps strength in COPD. Thorax. 2009;64(5):418-23.

5. Hermans G, Van Mechelen H, Clerckx B, Vanhullebusch T, Mesotten D, Wilmer A, et al. Acute outcomes and 1-year mortality of intensive care unit-acquired weakness. A cohort study and propensity-matched analysis. Am J Respir Crit Care Med. 2014;190(4):410-20.

6. Herridge MS, Cheung AM, Tansey CM, Matte-Martyn A, Diaz-Granados N, Al-Saidi F, et al. One-year outcomes in survivors of the acute respiratory distress syndrome. N Engl J Med. 2003;348:683-93. 
7. Fan E, Dowdy DW, Colantuoni E, Mendez-Tellez PA, Sevransky JE, Shanholtz C, et al. Physical complications in acute lung injury survivors: a two-year longitudinal prospective study. Crit Care Med. 2014;42(4):849-59.

8. van Gassel RJJ, Baggerman MR, van de Poll MCG. Metabolic aspects of muscle wasting during critical illness. Curr Opin Clin Nutr Metab Care. 2020;23(2):96-101.

9. Braunschweig CA, Sheean PM, Peterson SJ, Gomez Perez S, Freels S, Troy KL, et al. Exploitation of diagnostic computed tomography scans to assess the impact of nutrition support on body composition changes in respiratory failure patients. JPEN J Parenter Enteral Nutr. 2014;38(7):880-5.

10. Wei X, Day AG, Ouellette-Kuntz H, Heyland DK. The Association Between Nutritional Adequacy and Long-Term Outcomes in Critically III Patients Requiring Prolonged Mechanical Ventilation: A Multicenter Cohort Study. Crit Care Med. 2015.

11. Alberda C, Gramlich L, Jones N, Jeejeebhoy K, Day A, Dhaliwal R, et al. The relationship between nutritional intake and clinical outcomes in critically ill patients: results of an international multicenter observational study. Intensive Care Med. 2009;35:1728-37.

12. De Beaux I, Chapman M, Fraser R, Finnis M, De Keulenaer B, Liberalli D, et al. Enteral nutrition in the critically ill: A prospective survey in an Australian intensive care unit. Anaesth Intensive Care. 2001;29:619-22.

13. Chapman M, Peake SL, Bellomo R, Davies A, Deane A, Horowitz M, et al. Energy-Dense versus Routine Enteral Nutrition in the Critically III. N Engl J Med. 2018;379(19):1823-34.

14. Deane AM, Little L, Bellomo R, Chapman MJ, Davies AR, Ferrie S, et al. Outcomes Six Months after Delivering $100 \%$ or $70 \%$ of Enteral Calorie Requirements during Critical Illness (TARGET). A Randomized Controlled Trial. Am J Respir Crit Care Med. 2020;201(7):814-22.

15. Study protocol for the Augmented versus Routine Approach to Giving Energy Trial (TARGET). Crit Care Resusc. 2018;20(1):6-14.

16. Tillquist M, Kutsogiannis DJ, Wischmeyer PE, Kummerlen C, Leung R, Stollery D, et al. Bedside ultrasound is a practical and reliable measurement tool for assessing quadriceps muscle layer thickness. JPEN J Parenter Enteral Nutr. 2014;38(7):886-90.

17. Campbell IT, Watt T, Withers D, England R, Sukumar S, Keegan MA, et al. Muscle thickness, measured with ultrasound, may be an indicator of lean tissue wasting in multiple organ failure in the presence of edema. Am J Clin Nutr. 1995;62:533-9.

18. Chapple LS, Deane AM, Williams LT, Strickland R, Schultz C, Lange K, et al. Longitudinal changes in anthropometrics and impact on self-reported physical function after traumatic brain injury. Crit Care Resusc. 2017;19(1):29-36.

19. Ferrie S, Allman-Farinelli M, Daley M, Smith K. Protein Requirements in the Critically III: A Randomized Controlled Trial Using Parenteral Nutrition. JPEN J Parenter Enteral Nutr. 2016;40(6):795-805.

20. Ali NA, O'Brien JM, Hoffmann SP, Phillips G, Garland A, Finley JCW, et al. Acquired Weakness, Handgrip Strength, and Mortality in Critically III Patients. Am J Respir Crit Care Med. 2008;178(3):261-8. 
21. Mathias S, Nayak US, Isaacs B. Balance in elderly patients: the "get-up and go" test. Arch Phys Med Rehabil. 1986;67(6):387-9.

22. ATS statement: guidelines for the six-minute walk test. Am J Respir Crit Care Med. 2002;166(1):1117.

23. Chapple LS, Summers MJ, Weinel LM, Deane AM. Outcome Measures in Critical Care Nutrition Interventional Trials: A Systematic Review. Nutr Clin Pract. 2020;35(3):506-13.

24. Doig GS, Simpson F, Sweetman EA, Finfer SR, Cooper DJ, Heighes PT, et al. Early parenteral nutrition in critically ill patients with short-term relative contraindications to early enteral nutrition: a randomized controlled trial. JAMA. 2013;309(20):2130-8.

25. Ridley EJ, Davies AR, Parke R, Bailey M, McArthur C, Gillanders L, et al. Supplemental parenteral nutrition versus usual care in critically ill adults: a pilot randomized controlled study. Crit Care. 2018;22(1):12.

26. Wischmeyer PE, Hasselmann M, Kummerlen C, Kozar R, Kutsogiannis DJ, Karvellas CJ, et al. A randomized trial of supplemental parenteral nutrition in underweight and overweight critically ill patients: the TOP-UP pilot trial. Crit Care. 2017;21(1):142.

27. Baldwin $C E$, Bersten $A D$. Alterations in respiratory and limb muscle strength and size in patients with sepsis who are mechanically ventilated. Phys Ther. 2014;94(1):68-82.

28. Weinel LM, Summers MJ, Chapple LA. Ultrasonography to measure quadriceps muscle in critically ill patients: A literature review of reported methodologies. Anaesth Intensive Care. 2019;47(5):423-34.

29. Yk Yeo N, Aj Reddi B, Schultz CG, O'Connor SN, Chapman MJ, LA SC. Early anthropometry, strength, and function in survivors of critical illness. Aust Crit Care. 2021;34(1):33-7.

30. Santos LJd, Silveira FdS, Müller FF, Araújo HD, Comerlato JB, Silva MCd, et al. Avaliação funcional de pacientes internados na Unidade de Terapia Intensiva adulto do Hospital Universitário de Canoas. Braz J Phys Ther. 2017;24(4):437-43.

31. Solverson KJ, Grant C, Doig CJ. Assessment and predictors of physical functioning post-hospital discharge in survivors of critical illness. Ann Intensive Care. 2016;6(1):92.

32. Schuetz P, Fehr R, Baechli V, Geiser M, Deiss M, Gomes F, et al. Individualised nutritional support in medical inpatients at nutritional risk: a randomised clinical trial. Lancet. 2019;393(10188):2312-21.

33. Chapple LS, Deane AM, Heyland DK, Lange K, Kranz AJ, Williams LT, et al. Energy and protein deficits throughout hospitalization in patients admitted with a traumatic brain injury. Clin Nutr. 2016;35(6):1315-22.

34. Ridley EJ, Parke RL, Davies AR, Bailey M, Hodgson C, Deane AM, et al. What Happens to Nutrition Intake in the Post-Intensive Care Unit Hospitalization Period? An Observational Cohort Study in Critically III Adults. JPEN J Parenter Enteral Nutr. 2019;43(1):88-95.

35. Singer P, Blaser AR, Berger MM, Alhazzani W, Calder PC, Casaer MP, et al. ESPEN guideline on clinical nutrition in the intensive care unit. Clin Nutr. 2019;38(1):48-79. 
36. McClave SA, Taylor BE, Martindale RG, Warren MM, Johnson DR, Braunschweig C, et al. Guidelines for the Provision and Assessment of Nutrition Support Therapy in the Adult Critically III Patient: Society of Critical Care Medicine (SCCM) and American Society for Parenteral and Enteral Nutrition (A.S.P.E.N.). JPEN J Parenter Enteral Nutr. 2016;40(2):159-211.

37. Holwerda AM, Paulussen KJM, Overkamp M, Goessens JPB, Kramer IF, Wodzig W, et al. DoseDependent Increases in Whole-Body Net Protein Balance and Dietary Protein-Derived Amino Acid Incorporation into Myofibrillar Protein During Recovery from Resistance Exercise in Older Men. J Nutr. 2019;149(2):221-30.

38. Fetterplace K, Deane AM, Tierney A, Beach LJ, Knight LD, Presneill J, et al. Targeted Full Energy and Protein Delivery in Critically III Patients: A Pilot Randomized Controlled Trial (FEED Trial). JPEN J Parenter Enteral Nutr. 2018;42(8):1252-62.

39. Nakamura K, Kihata A, Naraba H, Kanda N, Takahashi Y, Sonoo T, et al. beta-Hydroxy-betamethylbutyrate, Arginine, and Glutamine Complex on Muscle Volume Loss in Critically III Patients: A Randomized Control Trial. JPEN J Parenter Enteral Nutr. 2020;44(2):205-12.

40. Viana MV, Becce F, Pantet O, Schmidt S, Bagnoud G, Thaden JJ, et al. Impact of beta-hydroxy-betamethylbutyrate (HMB) on muscle loss and protein metabolism in critically ill patients: A RCT. Clin Nutr. 2021;40(8):4878-87.

41. McNelly AS, Bear DE, Connolly BA, Arbane G, Allum L, Tarbhai A, et al. Effect of Intermittent or Continuous Feed on Muscle Wasting in Critical Illness: A Phase 2 Clinical Trial. Chest. 2020;158(1):183-94.

42. Paris MT, Mourtzakis M, Day A, Leung R, Watharkar S, Kozar R, et al. Validation of Bedside Ultrasound of Muscle Layer Thickness of the Quadriceps in the Critically III Patient (VALIDUM Study). JPEN J Parenter Enteral Nutr. 2017;41(2):171-80.

43. Puthucheary ZA, McNelly AS, Rawal J, Connolly B, Sidhu PS, Rowlerson A, et al. Rectus Femoris Cross-Sectional Area and Muscle Layer Thickness: Comparative Markers of Muscle Wasting and Weakness. Am J Respir Crit Care Med. 2017;195(1):136-8.

\section{Figures}




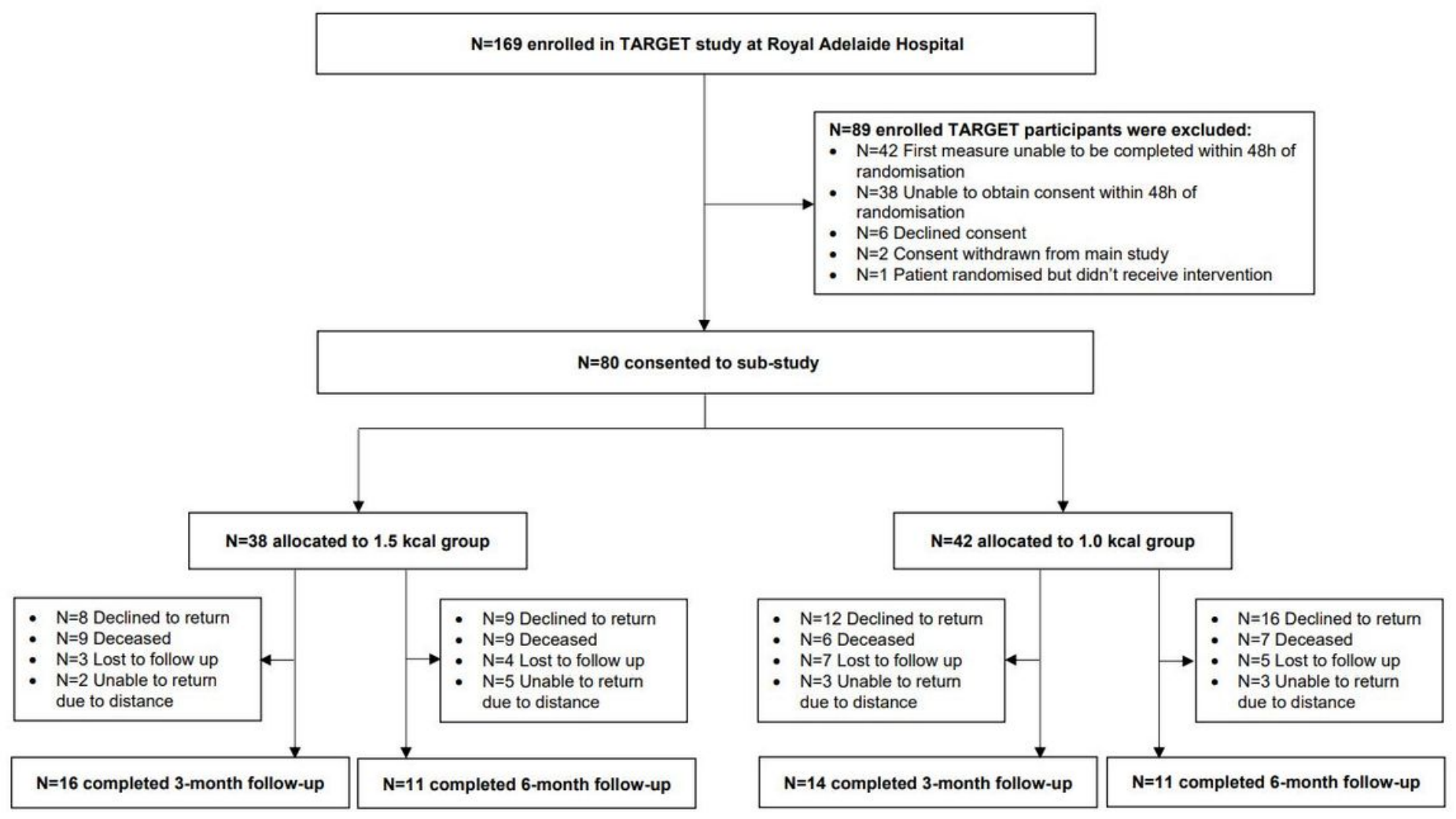

\section{Figure 1}

CONSORT diagram 


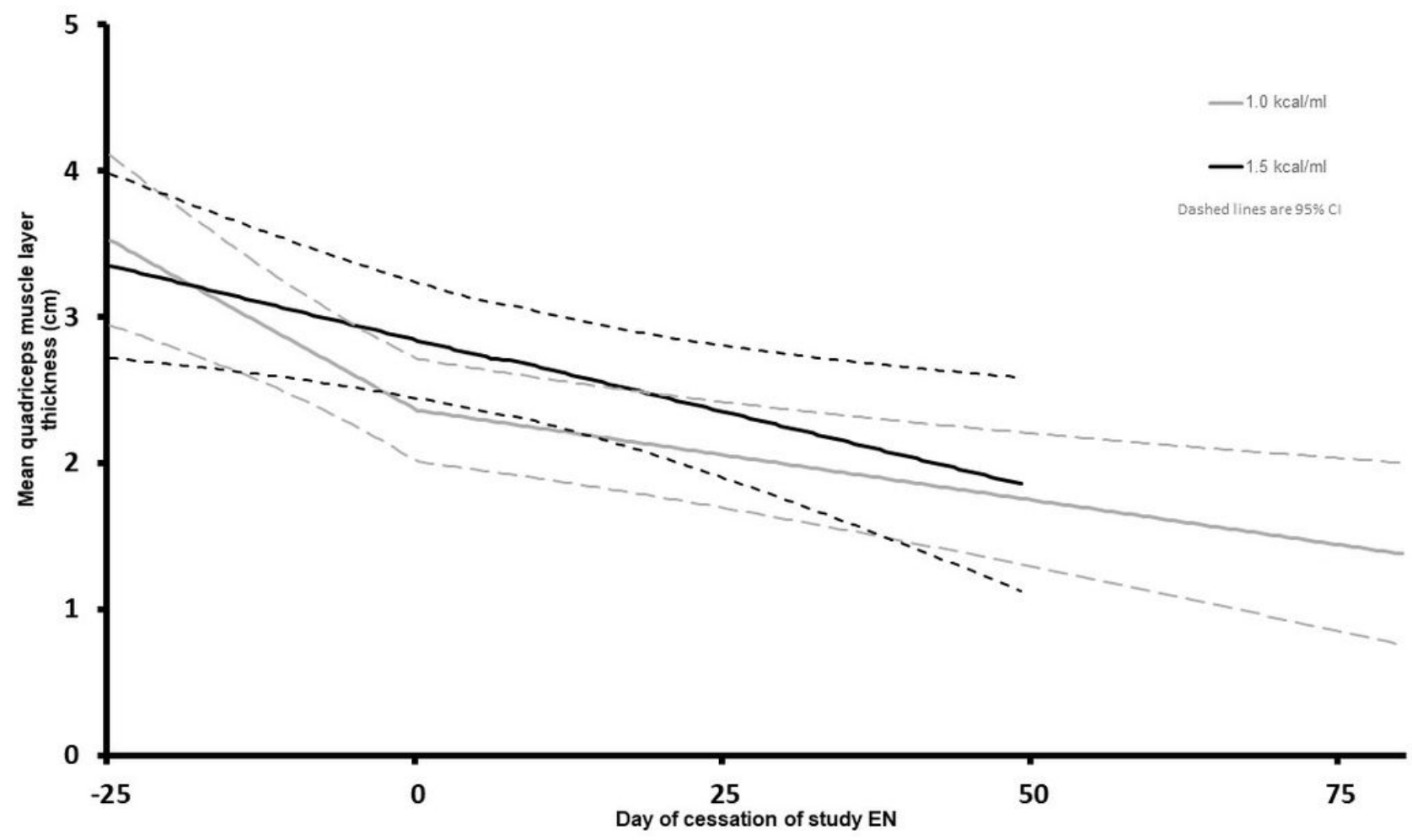

Figure 2

Changes in quadriceps muscle layer thickness, before and after ceasing study enteral nutrition 